vaginal birth, but caution should be taken in the first three minutes as oxygen saturation is higher.

\section{A COMPARISON OF NEONATAL BILIRUBIN DETERMINED BY BLOOD GAS ANALYZER AND AUTO ANALYZER}

doi:10.1136/archdischild-2012-302724.1742

K Obaid. Neonatology, Pediatrics, Diyala University Medical College, Baquba, Iraq

As high levels of serum bilirubin well known to be toxic to the brain especially preterm neonates, many methods adapted for bilirubin measurement. We evaluated total bedside blood gas analyzer bilirubin $(\mathrm{BsB})$ in preterm and term newborns compared to central laboratory auto analyzer bilirubin (TsB). An observational study on term and preterm newborns paired samples were checked for BsB and TsB looking for the significantly compared values, time needed, gestational age, weight, and anemia and blood volume extraction. A hundred forty one term and preterm newborns were included, birth weights range from 460 to $3450 \mathrm{~g}$ with a mean of 1450 and gestational ages from 23 to 40 weeks with a mean of 30.4 weeks. The correlation of $\mathrm{TsB}$ to BsB was linear and statistically significant, as well as hemoglobin and hematocrit, especially preterm neonate or term neonates already given phototherapy when the transcutaneous bilirubin measurement cannot be recommended the bedside blood gas analyzer bilirubin, hemoglobin and hematocrit were found to be statistically correlated and minimizing the time and extraction blood volume.

\section{EVALUATION OF NEONATAL COMPLICATIONS IN CAESARIAN SECTION AND VAGINAL DELIVERY}

doi:10.1136/archdischild-2012-302724.1743

'S Rahmani, 'R Saeedi, ${ }^{2} E$ Rahmani. 'Mashhad University of Medical Sciences, Mashhad; ${ }^{2}$ Hormozgan University of Medical Sciences, Gheshm, Iran

Background The appropriateness of the rising rate of cesarean delivery worldwide has been debated widely. U.S. cesareans have risen $40 \%$ since 1996 . IRAN cesareans section rate is $40 \_40 \%$ nowadays. However, the high rate of cesareans in the United States has not resulted in improved outcomes for babies or mothers. So must make a decision to lowered rate of SC with out risen of neonatal complications. The aim of this study is to evaluate neonatal complications in caesarian section and vaginal delivery.

Method We conducted observational study of all women with a singleton gestation and a prior cesarean delivery at 2 academic medical centers in Masshad medical university. Perinatal outcomes were compared between 770 neonates who had born with a normal vaginaly delivery and neonates who had born with an elective cesarean delivery without labor

Results Vaginal delivery was attempted by 344 women, and 426 women underwent elective cesarean delivery without labor. Asphyxia occurred in 30 infants whose mothers underwent elective cesarean delivery and in 11 infants born at term whose mothers underwent a trial of labor $(p<0.001)$. Planned cesarean delivery decreased rates of low up gar score from $11.2 \%$ to $17.8 \%(\mathrm{p}<0.001)$. The risk for pulmonary disorders (transient tachypnea of the newborn infant and respiratory distress syndrome) rose from $9 \%$ to $4.6 \%(p=00.001)$ in elective cesarean delivery.

Conclusion Fetal complications like RDS were significantly higher in cesarean section versus vaginal delivery.

\section{IS THERE A ROLE FOR A PART-TIME POISON CONTROL} CENTER IN A HUMAN RESOURCE LIMITED SETTING?

doi:10.1136/archdischild-2012-302724.1744

S Bharwani. Pediatrics, Faculty of Medicine and Health Sciences, United Arab Emirates University, FMHS, Al Ain, United Arab Emirates
Background and Aims The poison control centers have been shown to reduce the morbidity and mortality in childhood accidental poisoning. The United Arab Emirates poison and drug information center does not operate $24 / 7$ due to the shortage of well-trained staff. We aimed at identifying the time range of presentations of the poisoning cases to the emergency centers in the region as an indirect efficiency measure of the center.

Method We reviewed the electronic medical records for the documented triage check-in time for all the cases of accidental poison ingestion under the age of 10 years who presented in the year 2010 in the two tertiary emergency centers (ERs) in the region. We divided the 24 hour digital time clock into two distinct segments ie.0700 to 1500 hours (the working hours of the poison center in the region) and 1501 to 0659 hours (off working hours). The answers were compared using frequency bar plotting to get the percentage of children in each segment.

Results 200 children were seen between January $1^{\text {st }}$ and December $31^{\text {st }} 2010$ in the ERs. Record was not available in six cases. 153 cases (76.5\%) were checked-in in the 1501-0659 hours segment compared to the 41 cases $(20.5 \%)$ during the working hours of the poison center.

Conclusion More than three-quarter of accidental poison ingestion cases presented to the emergency when the poison center was closed. Shifting the day time staff to provide evening and night time coverage can significantly improve the efficiency of the poison center and minimize cost.

\section{THE DIAGNOSTIC CHALLENGE DURING THE PERINATAL PERIOD: ASYMPTOMATIC OVARIAN AUTOAMPUTATION}

doi:10.1136/archdischild-2012-302724.1745

HB Güvenç. Pediatric Surgery, Camlica Universal Hospital, Istanbul, Turkey

Background and Aims Initially defined as a rare complication, ovarian autoamputation is increasingly reported in neonates and infants less than one year of age. Antenatal ultrasound may not always be relevant in determining long term outcome of complex ovarian cysts. A sonographically persistent ovarian cyst may be free floating in the peritoneal cavity with no connection to the pelvis, or may parasite omental or mesenteric circulation, simulating an omental or mesenteric cyst.

Methods Five patients with prenatally diagnosed cystic masses are reported. The masses were initially detected during $28,33,34$ and 35 weeks of gestational age, with septations and dense echogenicity mimicking complex ovarian cysts. The cysts failed to resolve in all cases. Serum $\alpha$ FP levels were normal on date of admission in three elder cases and returned to normal in two newborns. $\beta$-hCG was within normal limits. CT or MRI studies confirmed cystic lesion with additional calcification area in two patients.

Results Laparoscopic approach revealed ovarian autoamputation in all patients with vessel resembling attachments originating from the greater omentum in two, and ileal mesenteric attachment in another. Histopathology revealed a hemorrhagic cyst with areas of infarction in four cases and locus of mature teratoma in one. Four patients were discharged within 24 hours postop.

Conclusions Radiology failed to detect torsion and autoamputation in all patients with prenatally diagnosed hypoechogenic cystic masses which failed to resolve following conservative management. The possibility of an otherwise silent ovarian autoamputation in a neonate, may necessitate laparoscopic intervention for correct diagnosis. COUGH IN THE COMIMUNITY

doi:10.1136/archdischild-2012-302724.1746 
'P Heinz, ${ }^{2}$ S Rann, 'R Ross Russell. 'Department of Paediatrics, Addenbrooke's Hospital; ${ }^{2}$ Shelford Medical Practice, Cambridge, UK

Introduction As economic pressures increase, many primary care providers are restricting referrals to hospital. Evidence based guidance for such restrictions are difficult to find and there is a risk that patients with significant disease may have their diagnoses delayed or missed. We have worked with colleagues in primary care to produce a single page guidance sheet for doctors seeing children with common (and occasionally important) symptoms.

Methods We started with a common problem - the coughing child. Most children can be managed in the community, but some have important underlying diseases that need more specialised investigation and treatment. We developed three tools

1. an evidenced algorithm for referral, clarifying which features of the history and examination indicated significant or worrying disease

2. recently published reviews that allowed professionals to read more on the subject,

3. good patient information that set out the principles of our advice.

These were reviewed and discussed at a joint meeting with primary care doctors and then the amended guidelines were disseminated.

Results The resultant guideline (one page with links to articles and information sheets) has been popular with primary care, and is available through the hospital website. It has been used in training. Involvement of primary care doctors has improved its credibility, and early analysis of referral patterns suggests that practices using the guidelines have a lower referral rate to secondary care than the local average, suggesting it modifies referral behaviour. We hope to employ the methodology in a wide range of common paediatric conditions.

\section{MATERNAL ACCESS TO COMMUNICATION IN HEALTH AND HEALTH CARE AND CHILDHOOD IMMUNISATION IN SELECTED PERI-URBAN SETTLEMIENTS IN KUMASI METROPOLIS, GHANA}

doi:10.1136/archdischild-2012-302724.1747

D Buor. Valley View University, Accra, Ghana

The paper examines the effects of mass media health communication, specifically, maternal access and use of media communication in health and health care on childhood immunisation at the periurban settlements in the Kumasi metropolis. A sample of 240 mothers, drawn using random (chance selection) was used for the survey. The main research instrument was formal interview (face-to-face). Charts were used to depict the results whilst chi square tests, derived from cross-tabulation using the Statistical Package for Social Scientists (SPSS), were used to determine significant differences among the independent variables. Results show that children whose parents regularly access mass media health communication go through all or greater part of immunisation methods. Other factors that influence childhood immunisation are maternal age, schooling, employment, marital status and husband's education. For maternal access of health communication, the significant factors influencing it are maternal education, husband's education and marital status. Recommendations made to improve maternal access of childhood immunisation facilities and health communication include, compulsory education of the girl-child beyond basic education, informal education of illiterate mothers, provision of mass media health communication facilities such as television sets in the communities, enhancement of health communication at the hospitals, the simplification of the language used in health communication, and the regular use of local languages. Further research areas have been proposed.
1748 OPEN ACCESS TO HOSPITAL: A WAY TO IMPROVE CARE OF CHILDREN WITH CHRONIC CONDITIONS

doi:10.1136/archdischild-2012-302724.1748

H Bentur, SP Hulikere. Paediatrics, Warrington and Halton Hospitals NHS Foundation Trust, Warrington, UK

Background and Aims Children with chronic conditions have complex healthcare needs. They need a service which ensures easy access with continuity of care while providing co-ordination and support for self-management. Open access to hospital provides one such service and we reviewed our existing local practice to gain insight and improve our service.

Methods Study was conducted at Warrington General Hospital, which is a busy district general hospital in United Kingdom. This is a retrospective review of patients who have an open access to children's ward. Patient information regarding the lead consultant, diagnosis, management plan and advice available from open access folder were reviewed.

Results Total number of patients with open access to children's ward were $182.95 \%$ had information regarding their diagnosis, $80 \%$ had named lead consultant. Only 35\% had information on advice and management, and $30 \%$ had information regarding patient medication. There was overall poor documentation and poor information on patient details. It was also noted that there was no consistency by the consultants regarding the layout of the open access letter.

Conclusion Patient information and management plan were incomplete in majority. Our data shows the need to identify these children with chronic complex needs who can access hospital easily, have a system to keep up-to date information which will help in provision of better care and service, and share information among different professionals.

Based on these findings, we have made recommendations to improve the local practice and information management.

\section{SAFETY NETTING INFORMATION FOR THE ACUTELY SICK CHILD: SYSTEMATIC LITERATURE ON EFFECTIVENESS}

doi:10.1136/archdischild-2012-302724.1749

${ }^{1} \mathrm{M}$ Lakhanpaul, ${ }^{2} \mathrm{~S}$ Neill, ${ }^{2} \mathrm{C}$ Shang, ${ }^{3} \mathrm{M}$ Thompson, on behalf of the ASK SNIFF Team. ${ }^{1}$ General and Adolescent Paediatric Unit, University College London, Institute of Child Health, London; 'University of Northampton, Northampton; ${ }^{3 P r i m a r y}$ Care Health Sciences, University of Oxford, Oxford, UK

Background and Aims Uncertainty and anxiety surround parents' decisions to seek medical help for a sick child at home because parents are concerned not to miss serious illness whilst also not bothering the doctor unnecessarily. This dilemma leads to parents' desire for more information to support their decision making. Factors influencing the success of information resources need to be identified prior to the development of safety netting interventions for families. The ASK SNIFF team (Acutely Sick Kid Safety Netting Interventions for Families) aimed to review the literature on the effectivenss of existing resources to provide an evidence base for the develoment of safety netting information for parents to be used when determining to seek help for an acutely sick child.

Methods The initial stage of the project involved a systematic review using narrative analysis to identify influences on the effectiveness of information resources for parents caring for an acutely ill child at home.

Results A range of measures for effectiveness have been used in the literature which limits their comparison. Interventions that included information on more than one illness or symptom were found to be more effective. Parents were more likely to act on information provided to them in the community than in the emergency 